\title{
Sobre el empleo de las categorías "elemento compositivo" y "prefijo" en los diccionarios de la RAE
}

\author{
Marta Torres Martínez \\ Universidad de Jaén, España*
}

\begin{abstract}
Resumen
En este trabajo analizamos, valoramos e interpretamos el tratamiento de los prefijos en una selección de obras gramaticales y lexicográficas publicadas por la Real Academia Española a lo largo de los siglos XVIII, XIX y XX. Nuestro punto de partida es el Diccionario de la lengua española (DRAE 2001), en que hemos observado cómo los prefijos incluidos en una lista preexistente (Varela y Martín García 1999) son catalogados bajo dos categorías gramaticales distintas: 'prefijo' y 'elemento compositivo'. El objetivo principal es determinar por qué unidades de igual naturaleza, tales como inter- y super(procedentes de preposiciones latinas, INTER y SUPER, también prefijos en dicha lengua), son denominadas de manera diferente en DRAE (2001): 'prefijo' y 'elemento compositivo', respectivamente. Para ello, además de analizar los artículos lexicográficos relativos a estas dos etiquetas gramaticales, presentamos una revisión histórica del tratamiento categorial que recibe una selección de prefijos de similar índole en diferentes ediciones del diccionario académico
\end{abstract}

*Para correspondencia, dirigirse a: Marta Torres Martínez (matorma@ujaen.es), Departamento de Filología Española, Facultad de Humanidades y Ciencias de la Educación, Campus de las Lagunillas, s/n, 23071, Jaén, España. 
(Diccionario de autoridades, 1726-39 y DRAE 1803, 1884, 1925 y 1970). Por otra parte, creemos fundamental comprobar la información morfológica que ofrece la obra gramatical académica. Así, revisamos brevemente el tratamiento de los prefijos en las Gramáticas de la Real Academia Española (GRAE) publicadas entre 1771 y 1931 y, además, examinamos las ideas contenidas tanto en el Esbozo de una nueva gramática de la lengua española (1973) como en la Nueva gramática de la lengua española de la Academia (NGRAE 2009). En definitiva, nos interesa profundizar en la delimitación prefijo/tema culto y en su codificación tanto en la producción lexicográfica como en la obra gramatical de la Real Academia Española.

Palabras clave: prefijo, elemento compositivo, diccionario, gramática, Real Academia Española.

\title{
ON THE USE OF THE 'COMPOUND ELEMENT' AND 'PREFIX' CATEGORIES IN THE RAE DICTIONARIES
}

\begin{abstract}
In this article we analyse, assess and interpret how prefixes have been treated in some selected grammatical and lexicographic works published by the Real Academia de la Lengua throughout the 18th, 19 th and 20th centuries. Our starting point is the Diccionario de la lengua española (DRAE 2001), which classifies the prefixes extracted from a previous study (Varela and Martín García 1999) under two major grammatical categories: 'prefix' and compounding element'. We aim at determining why units of an apparently similar nature are labelled in two different ways in DRAE (2001): 'prefixes' and 'composite / compounding' elements (a case in point is that of the prefixes 'inter-' and 'super-', which come from the Latin prepositions INTER and SUPER, also described as prefixes). Besides, this study briefly reviews the historical categorial treatment given to a selection of prefixes in from several editions of this academic dictionary (Diccionario de autoridades, 1726-39, and DRAE 1803, 1884, 1925 and 1970). Finally, it is our belief that a morphological description is essential for an accurate grammatical description, we additionally offer an overview of the treatment of prefixes in the grammars of the Real Academia de la Lengua (GRAE) published between 1771 and 1931. The present study also examines the ideas underlying the Esbozo de una nueva gramática de la lengua española (1973) and the Nueva gramática de la lengua española (NGRAE 2009).
\end{abstract}


Key words: prefix, compounding element, dictionary, grammar, Royal Spanish Academy.

Recibido: 31/08/10. Aceptado: 04/05/11.

\section{INTRODUCCIÓN}

Nuestra investigación se centra en el tratamiento lexicográfico que reciben en el Diccionario de la lengua española de la Real Academia Española (DRAE 2001) las unidades que actualmente denominamos prefijo. Particularmente, nos interesa estudiar la categoría gramatical que este diccionario asigna a los 93 prefijos incluidos en una lista preexistente, la propuesta por Varela y Martín García en la Gramática descriptiva de la lengua española (1999).

En concreto, pretendemos determinar la razón por la que a unidades de igual índole, tales como inter- y super- (procedentes de preposición latina, INTER y SUPER, además de prefijos en dicha lengua, INTER- e SUPER-), se les asignan categorías diferentes en el DRAE (2001), "prefijo" y "elemento compositivo", respectivamente. Para ello, además de analizar los artículos lexicográficos relativos a estas dos etiquetas gramaticales, presentamos una revisión histórica del tratamiento categorial que recibe una selección de prefijos de similar naturaleza en diferentes ediciones del diccionario académico (Diccionario de autoridades, 1726-39 y DRAE 1803, 1884,1925 y 1970).

Además, creemos fundamental comprobar la información morfológica que ofrece la obra gramatical académica. Por este motivo, ofrecemos una breve revisión del tratamiento de los prefijos en las Gramáticas de la Real Academia Española (GRAE) publicadas entre 1771 y 1931 y, además, examinamos las ideas contenidas tanto en el Esbozo de una nueva gramática de la lengua española (1973) como en la Nueva gramática de la lengua española de la Academia (NGRAE 2009).

En definitiva, nos interesa profundizar en la delimitación prefijo/tema culto y en su codificación tanto en la producción lexicográfica como en la obra gramatical de la Real Academia Española. 


\section{TRATAMIENTO DE LOS PREFIJOS EN LOS DICCIONARIOS ACADÉMICOS}

\subsection{Punto de PARTIDA: DRAE (2001)}

Como hemos indicado en la introducción, partimos del inventario de prefijos propuesto por Varela y Martín García en la Gramática descriptiva de la lengua española, publicada en 1999 y dirigida por los profesores Ignacio Bosque y Violeta Demonte ${ }^{1}$ :

a-, ab-, ambi-, anfi-, ante-, anti-, apo-, archi-, auto-, bi-, bien-, casi-, centi-, circun-, cis-, citra-, con-, contra-, cuatri-, deca-, deci-, des-, dia-, dodeca-, ecto-, en-, endeca-, endo-, enea-, entre-, epi-, equi-, ex-, exo-, extra-, fuera-, hecto-, hemi-, hepta-, hetero-, hexa-, hiper-, hipo-, homo-, in-, infra-, inter-, intra-, intro-, iso-, macro-, mal-, maxi-, medio-, mega-, meta-, micro-, mili-, mini-, mono-, multi-, neo-, no-, octa-, octo-, paleo-, para-, penta-, per-, peri-, pluri-, poli-, post-, pre-, pro-, re-, recién-, retro-, semi-, seudo-, sex-, sin-, sobre-, sub-, super-, supra-, tetra-, todo-, trans-, tri-, ultra-, uni-, vice-.

Tras revisar la codificación lexicográfica de las unidades en el $D R A E$ (2001) y atender especialmente a la categoría gramatical asignada en cada caso, ofrecemos a continuación los datos observados:

i. Algunos elementos son denominados "prefijo": a-, anti-, cis-, con-, des-, dia-, en-, entre-, epi-, ex-, extra-, in-, inter-, intra-, para-, per-, peri-, post-, pre-, pro-, re-, sin-, sub-, trans-.

ii. La mayoría de las unidades es catalogada con el término "elemento compositivo": anfi-, ante-, archi-, auto-, bi-, centi-, circun-, cuatri-, deca-, deci-, ecto-, endeca-, endo-, enea-, equi-, hecto-, hemi-, hepta-, hetero-, hexa-, hiper-, hipo-, homo-, infra-, iso-, macro-, maxi-, mega-, meta-, micro-, mili-, mini-, mono-, multi-, neo-, paleo-, penta-, pluri-,

1 A propósito de la falta de unanimidad al establecer el inventario de prefijos del español, vid. Felíu (2003: 41). La elección de la nómina manejada en nuestra investigación se debe a que, a diferencia de otras propuestas, Varela y Martín García (1999) siguen unos criterios claros y precisos de carácter sincrónico, distribucional, formal y funcional. 
poli-, retro-, semi-, seudo-, sobre-, super-, supra-, tetra-, tri-, ultra-, vice-.

iii. Hallamos un tratamiento especial en el caso de bien- y contra-: ambas unidades son lematizadas sin guion -elemento ortográfico que indica el carácter ligado de los prefijos-y ninguna de ellas es denominada "prefijo" o "elemento compositivo", sino "adverbio [...] casi a manera de prefijo" y "preposición [...] en voces compuestas u. c. prefijo", respectivamente:

bien (Del lat. bene, bien) [...] adv. m. U. con algunos participios, casi a manera de prefijo, llegando a veces a formar con ellos una sola palabra. Bien criado, Bien hablado. (DRAE 2001, s. v. bien).

contra (Del lat. contra) 1. prep. Denota la oposición y contrariedad de una cosa con otra. En voces compuestas, u. c. pref. Contrabando, contraponer, contraveneno. [...] (DRAE 2001, s. v. contra).

iv. En el $D R A E$ (2001) no se incluyen las siguientes unidades: $a b-$, ambi-, apo-, casi-, citra-, dodeca-, exo-, fuera-, intro-, mal- (adj.), medio(adj.), no-, octa-, octo-, recién-(adv.), sex-, todo- (adj.), uni-.

En (3) ofrecemos un gráfico en el que se ilustra la información presentada anteriormente:

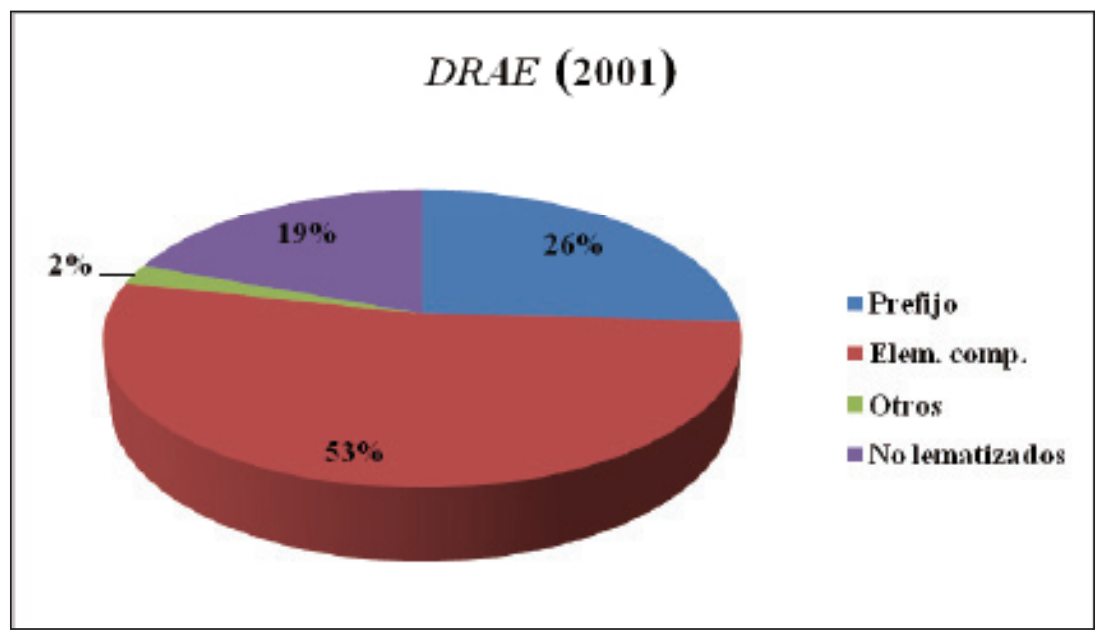


Podemos observar cómo un 19\% de las unidades incluidas en la lista de prefijos de la que partimos no se incorporan al DRAE (2001). En lo que respecta a las unidades de nuestra inicial lematizadas en la última edición del diccionario académico, el 53\% es codificado bajo la categoría gramatical de "elemento compositivo", frente al $26 \%$ de las unidades, que son denominadas "prefijo". Además, cabe destacar que solo dos elementos quedan caracterizados mediante otra categoría ("adverbio" y "preposición" en el caso de bien- y contra-), como ya hemos indicado anteriormente.

En definitiva, a la vista del tratamiento que el DRAE (2001) concede a los prefijos incluidos en la nómina de Varela y Martín García (1999), llaman la atención de manera especial las cuestiones que planteamos seguidamente:

i. La lista de unidades consideradas "elementos compositivos" duplica la nómina de las denominadas "prefijos" (53\% y 26\%, respectivamente).

ii. Elementos que poseen índole similar son definidos mediante categorías diferentes. Por ejemplo, sub- queda caracterizado como "prefijo", mientras que infra- es llamado "elemento compositivo", si bien ambos comparten un origen etimológico similar (proceden de preposición latina, SUB e INFRA, y se documentan ya como prefijos en dicha lengua, SUB- e INFRA-) y, además, poseen el mismo valor semántico ('debajo').

iii. El DRAE (2001) recoge elementos como endeca-, pero no incluye dodeca-. Igualmente, no se lematiza octo-, aunque se documentan otros de igual naturaleza como hepta-, hexa- y penta-. De otro lado, en el diccionario se describe el uso como prefijo de bien-, aunque no hallamos información alguna sobre el empleo ligado de mal-.

iv. Al quedar definidos mediante las categorías "adverbio" y "preposición”, la Academia no lematiza bien- y contra- seguidos de guion.

A fin de revelar la razón por la que el $D R A E$ (2001) opta por emplear las categorías gramaticales "elemento compositivo" y "prefijo" al caracterizar las unidades objeto de estudio, analizamos seguidamente los artículos correspondientes a sendos lemas:

prefijo, ja. (Del lat. praefixus, part. de praefigere, colocar delante.) adj. Gram. Dicho de un afijo: Que va antepuesto: p. ej., en desconfiar, reponer. U. t. c. s. m. (DRAE 2001: s. v. prefijo).

afijo, ja. (Del part. irreg. ant. de afjar) adj. Gram. Se dice de los prefijos, infijos y sufijos. U. m. c. s. m. (DRAE 2001: s. v. prefijo). 
elemento. $[\ldots] \| \sim$ compositivo. m. Gram. Componente, no independiente, por lo general de origen griego o latino, que interviene en la formación de palabras compuestas, anteponiéndose o posponiéndose a otro. En los encabezamientos de los artículos correspondientes, este Diccionario los registra seguidos de guion si inician el compuesto, y precedidos de guion si se posponen a otro u otros morfemas; p. ej., auto-, fono-, -fono, grafo-, -grafo. (DRAE 2001: s. v. elemento).

En (4) observamos que, por un lado, existen similitudes al definir ambos lemas: (i) la Academia emplea un criterio colocacional o posicional: el prefijo "va antepuesto", mientras que los elementos compositivos pueden "anteponerse o posponerse"; (ii) además, comprobamos que el elemento compositivo queda caracterizado como un "componente no independiente", caracterización a la que también responden los “afijos”, categoría mediante la que se identifica al prefijo. Por otro lado, hay que destacar que en el artículo correspondiente al lema elemento compositivo se indica el origen etimológico de este tipo de unidades ("griego o latino"), información que se omite en el caso del lema prefijo - no obstante, las palabras complejas propuestas como ejemplos son creadas a partir de los prefijos de origen latino, des-y re--.

Tras analizar los artículos lexicográficos de prefijo y elemento compositivo advertimos que ambas unidades comparten características tales como su condición ligada y la posición que ocupan en la palabra compleja -aunque los "elementos compositivos" constituyen a veces el segundo segmento de la unidad léxica como en el caso de -fono o -grafo, la Academia también incluye entre los ejemplos auto-, elemento que solo puede anteponerse a la base léxica. De este modo, en el diccionario parece que no encontramos la clave a partir de la que podamos distinguir claramente un "prefijo" de un "elemento compositivo", pues las dos categorías pueden emplearse a la hora de caracterizar las unidades de la nómina de Varela y Martín García (1999).

No obstante, aunque, a la vista de los artículos lexicográficos de (4) nos parezca que la Academia emplea aleatoriamente las categorías de "prefijo" y "elemento compositivo", si observamos la lista de unidades llamadas de una y otra manera, nos percatamos de que el motivo del dispar tratamiento radica en el origen categorial grecolatino de las unidades en cuestión.

En efecto, la mayoría de las unidades denominadas "prefijo" proceden de preposición latina o griega y ya se documentan como prefijos en tales lenguas (anti-, cis-, con-, dia-, en-, entre-, epi-, ex-, extra-, in-, inter-, intra-, para-, per-, peri-, post-, pre-, pro-, sin-, sub-, trans-). Solo encontramos cuatro casos que poseen una etimología distinta, a saber, no se documentan como preposiciones en sus lenguas de origen, sino exclusivamente como prefijos, $a$-, des-, in- negativo y re-. Además, hay que destacar que los prefijos con-, 
en-y entre- coinciden formalmente con preposiciones del español (con, en y entre, respectivamente).

De otra parte, la mayoría de las unidades catalogadas como "elementos compositivos" no posee origen preposicional (archi-, auto-, bi-, centi-, cuatri-, deca-, deci-, ecto-, endeca-, endo-, enea-, equi-, hecto-, hemi-, hepta-, hetero-, hexa-, homo-, iso-, macro-, maxi-, mega-, micro-, mili-, mini-, mono-, multi-, neo-, paleo-, penta-, pluri-, poli-, retro-, semi-, seudo-, tetra-, tri-, vice-), a excepción de anfi-, ante-, circun-, hiper-, hipo-, infra-, meta-, sobre-, super-, supra- y ultra-, prefijos con correlato preposicional en sus lenguas de origen.

De este modo, parece que la Academia sigue el criterio etimológico, salvo en el caso de las excepciones observadas, a la hora de caracterizar las unidades objeto de estudio, y denomina generalmente "prefijo" a los morfemas que proceden de preposición grecolatina y "elemento compositivo" a aquellos que poseen distinto origen categorial.

\subsection{BREVE REVISIÓN HISTÓRICA: DiCCIONARIO DE AUTORIDADES (1726-39) y DRAE 1803, 1884, 1925 Y 1970}

A continuación, nos parece interesante revisar el tratamiento lexicográfico de algunos prefijos en una selección de diccionarios académicos a fin de observar cómo se trata la delimitación prefijo/tema grecolatino en siglos anteriores.

En cuanto a los prefijos seleccionados, hemos considerado ocho unidades organizadas en parejas atendiendo a su carácter etimológico y categorial:

i. De un lado, analizamos circun-, peri- y anti-, contra-, cuatro prefijos que proceden y coinciden formalmente con una preposición grecolatina, y además ya se documentan como prefijos en sus lenguas de origen.

ii. De otro lado, revisamos equi- e iso-, unidades que no proceden de preposición, sino de otras clases de palabra y que ya funcionan como prefijos en la tradición grecolatina.

iii. Finalmente, estudiamos bi-y tetra-, que solo funcionan como prefijos en la lengua de origen.

Además de agrupar los prefijos según su origen categorial, nos interesa igualmente comparar aquellos que comparten valor semántico, siguiendo los criterios establecidos en la $N G R A E$ (2009: $§ 10)$ : 
i. Prefijos de sentido locativo ('movimiento o posición alrededor') en el caso de circun- y peri-

ii. Prefijos de orientación o disposición opositiva en el caso de anti- y contra-

iii. Prefijos adjetivales que denotan igualdad en el caso de equi- e iso-

iv. Prefijos cuantificativos, asociados con la noción de cantidad o número en el caso de bi- y tetra-

Además, en lo que respecta a su etimología, cada pareja de prefijos queda constituida por uno de origen griego y otro de origen latino.

De otra parte, en lo concerniente al corpus lexicográfico, además del DRAE (2001), los cuatro diccionarios manejados representan distintas etapas de la historia de la lexicografía académica:

i. Como es sabido, el Diccionario de autoridades (DA 1726-39) es el primer repertorio publicado por la RAE, del que beberán todas las ediciones del diccionario usual que se han publicado hasta el momento.

ii. El DRAE (1803) supone la primera edición del diccionario usual publicada en el siglo XIX.

iii. El $D R A E$ publicado en 1884 es la penúltima edición publicada a finales del siglo XIX, interesante por las novedades que introduce en relación con las ediciones anteriores: (i) la introducción de etimologías, en lugar de las correspondencias latinas suprimidas en la edición anterior (DRAE 1869); (ii) la acogida de palabras pertenecientes al léxico científico-técnico, (iii) la inclusión y revisión de artículos relativos a lemas del lenguaje literario y vulgar, (iv) el aumento de diminutivos $\mathrm{y}$ aumentativos en la macroestructura del repertorio ${ }^{2}$ y (v) la revisión de la marca "anticuada".

iv. Además de ser elegida para que represente la etapa relativa a la primera mitad del siglo XX, la edición de 1925 ha sido consultada

2 Si bien se indica que "se han suprimido todos los de estas vulgares desinencias [ico, illo, ito y en on y azo], como igualmente los superlativos en ísimo [...] porque las reglas de su formación, que pueden y deben estudiarse en la Gramática, irán, a mayor abundamiento, como apéndice del Diccionario" (DRAE, 1884: VI). Hemos de señalar que las Reglas para la corrección y aumento del Diccionario vulgar redactadas en 1870 contienen los mismos criterios que las publicadas en 1869 en lo tocante al tratamiento de los "compuestos" y "derivados". 
porque en ella documentamos por vez primera el término "prefijo" como categoría principal (en el caso del lema in- negativo) ${ }^{3}$.

v. El DRAE (1970) se ha seleccionado por dos motivos esenciales: se trata de una de las ediciones publicadas a lo largo de la segunda mitad del siglo XX (junto al DRAE 1956, 1984 y 1992) y en ella documentamos por vez primera el término "elemento compositivo".

A continuación, analizamos la categoría gramatical asignada a los prefijos seleccionados en cada uno de los repertorios lexicográficos objeto de estudio:

\begin{tabular}{|c|c|c|c|c|c|c|}
\hline \multicolumn{7}{|c|}{$\begin{array}{l}\text { Unidades que proceden y coinciden formalmente con una preposición } \\
\text { en latín y griego y ya se documentan como prefijos en tales lenguas }\end{array}$} \\
\hline & $\begin{array}{c}D A \\
(1726-39)\end{array}$ & $\begin{array}{c}D R A E \\
(1803)\end{array}$ & $\begin{array}{c}D R A E \\
(1884)\end{array}$ & $\begin{array}{c}D R A E \\
(1925)\end{array}$ & $\begin{array}{c}D R A E \\
(1970)\end{array}$ & $\begin{array}{c}D R A E \\
(2001)\end{array}$ \\
\hline $\begin{array}{l}\text { circun- } \\
\text { (Del lat. } \\
\text { circum-) }\end{array}$ & \multicolumn{2}{|l|}{$\varnothing$} & \multicolumn{3}{|c|}{$\begin{array}{l}\text { preposición inseparable } \\
\text { (sin guion) }\end{array}$} & $\begin{array}{l}\text { elemento } \\
\text { compositivo }\end{array}$ \\
\hline $\begin{array}{l}\text { peri- } \\
\text { (Del gr. } \\
\pi \varepsilon \rho l-)\end{array}$ & \multicolumn{2}{|l|}{$\varnothing$} & \multicolumn{3}{|c|}{$\begin{array}{l}\text { preposición inseparable } \\
\text { (sin guion) }\end{array}$} & prefijo \\
\hline
\end{tabular}

Prefijos de sentido locativo ('movimiento o posición alrededor')

Circun- queda lematizado en el $D R A E$ a partir de 1884, bajo la categoría "preposición inseparable" -término empleado de manera habitual para catalogar los prefijos a lo largo del siglo XIX- y sin guion, elemento que se incorpora a partir del DRAE (1984). Ya en 1992 se denomina "elemento compositivo".

En el caso de peri-, al igual que circun- es incluido en el diccionario a partir de 1884 bajo la categoría de "preposición inseparable" y sin guion,

\footnotetext{
3 Además, la edición del $D R A E$ publicada en 1925 destaca en la historia de la lexicografía académica debido a que la Corporación modifica el título de la obra, pues sustituye el adjetivo "castellana" por "española" (Diccionario de la lengua española).
} 
que se incorpora también en 1984. En el DRAE (1992) cambia de categoría gramatical, de "preposición inseparable" a "prefijo".

Observamos que, si bien estos dos prefijos poseen una trayectoria similar a lo largo de los siglos XVIII, XIX y XX, en la nueva edición del diccionario académico presentan categorías gramaticales diferentes.

\begin{tabular}{|c|c|c|c|c|c|c|}
\hline \multicolumn{7}{|c|}{$\begin{array}{l}\text { Unidades que coinciden formalmente con una preposición en latín y } \\
\text { griego y ya se documentan como prefijos en tales lenguas }\end{array}$} \\
\hline & $\begin{array}{c}D A \\
(1726-39)\end{array}$ & $\begin{array}{c}D R A E \\
(1803)\end{array}$ & $\begin{array}{c}D R A E \\
(1884)\end{array}$ & $\begin{array}{c}D R A E \\
(1925)\end{array}$ & $\begin{array}{c}D R A E \\
(1970)\end{array}$ & $\begin{array}{c}D R A E \\
(2001)\end{array}$ \\
\hline $\begin{array}{l}\text { anti- } \\
\text { (Del gr. } \\
\text { ávtl-) }\end{array}$ & $\varnothing$ & preposi & $\begin{array}{l}\text { ción inse } \\
\text { sin guion }\end{array}$ & parable & $\begin{array}{l}\text { elemento } \\
\text { compositivo }\end{array}$ & prefijo \\
\hline $\begin{array}{l}\text { contra- } \\
\text { (Del lat. } \\
\text { contra) }\end{array}$ & \multicolumn{2}{|l|}{ Ø } & \multicolumn{4}{|c|}{$\begin{array}{l}\text { prep. }[\ldots] \text { tiene uso como prefijo en voces } \\
\text { compuestas (sin guion) }\end{array}$} \\
\hline
\end{tabular}

Prefijos de significado de orientación o disposición ('oposición')

Anti- es lematizado en una edición temprana, la de 1803. La categoría gramatical que se le asigna hasta 1956 es "preposición inseparable". A partir de 1970 se le añade el guion y se lo denomina "elemento compositivo", si bien en 1992 se produce un cambio categorial y se lo define bajo el término "prefijo".

En lo tocante a contra-, aunque ya en latín se documenta como prefijo (CONTRADICO, 'contradecir'; CONTRAJURIS, 'contrario a la ley'), su empleo como elemento ligado en español no se reconoce hasta la edición de 1884. En la actualidad sigue sin codificarse no seguido de guion y bajo la categoría de "preposición". No obstante, hemos comprobado que desde el Diccionario de autoridades se documentan palabras complejas formadas a partir de este prefijo, tales como las recogidas en (7):

ContraArmiños. s. m. Término del Blasón. Color contrario al armiño, esto es, campo negro con moscas blancas. (DA 1729: s. v. contraarmiños).

ContraAtaques. s. m. son unas líneas como los ataques que se hacen de noche y con el secreto posible, por las quales los de una 
plaza sitiada puedan, sin ser vistos, acercarse al enemigo. ( $D A$

1729: s. v. contraataques).

Contrabatería. s. f. Batería en oposición de otra. Es voz compuesta de la preposición Contra y del nombre Batería. (DA 1729: s. v. contrabatería).

Contraorden. s. m. el orden que se da contra el que antecedentemente estaba dado. (DA 1729: s. v. contraorden).

De este modo, si comparamos la evolución de anti-y contra-, podemos observar que existe un tratamiento lexicográfico bastante dispar, a pesar de que ambos prefijos poseen el mismo origen categorial.

\begin{tabular}{|l|c|c|c|c|c|c|}
\hline \multicolumn{6}{|c|}{$\begin{array}{l}\text { Unidades que en la tradición grecolatina ya funcionan como prefijos, si } \\
\text { bien no coinciden con preposiciones, sino con otras clases de palabras }\end{array}$} \\
\hline & $\begin{array}{c}D A \\
(1726-39)\end{array}$ & $\begin{array}{c}D R A E \\
(1803)\end{array}$ & $\begin{array}{c}D R A E \\
(1884)\end{array}$ & $\begin{array}{c}D R A E \\
(1925)\end{array}$ & $D R A E(1970)$ & $\begin{array}{c}D R A E \\
(2001)\end{array}$ \\
\hline $\begin{array}{l}\text { equi- } \\
\text { (Del lat. } \\
\text { aequi-) }\end{array}$ & \multicolumn{2}{|c|}{$\varnothing$} & $\begin{array}{c}\text { partícula pre- } \\
\text { positiva }\end{array}$ & $\begin{array}{c}\text { partícula } \\
\text { inseparable }\end{array}$ & $\begin{array}{c}\text { elemento com- } \\
\text { positivo }\end{array}$ \\
\hline $\begin{array}{l}\text { iso- } \\
\text { (Del gr. } \\
\text { ío-) }\end{array}$ & \multicolumn{7}{|c|}{$\varnothing$} & \multicolumn{2}{|c|}{ elemento compositivo } \\
\hline
\end{tabular}

Prefijos adjetivales que denotan igualdad

Equi- (procede de AEQUUS, 'igual', AEQUǏFORMIS, 'de igual forma') se incluye en el DRAE (1884), en esta edición es denominado "partícula prepositiva"; a partir de 1956 se denominará "partícula inseparable", mientras que ya en 1992 pasa a ser denominado "elemento compositivo" y tal categoría se mantiene en el DRAE (2001).

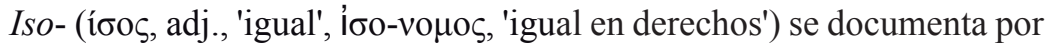
vez primera en el DRAE (1970) bajo la categoría "elemento compositivo" $\mathrm{y}$, al igual que equi-, la conserva en 1992 y 2001.

Llama la atención que la Academia no asigne la categoría "elemento compositivo" a equi- en el DRAE (1970), pues ya en esta edición se comienza a emplear tal término para caracterizar unidades como iso-. Igualmente, es relevante la tardía introducción en la macroestructura de iso- (DRAE 1970), si tenemos en cuenta que equi- se incluye en el último cuarto del siglo XIX. 
(9)

\begin{tabular}{|c|c|c|c|c|c|c|}
\hline \multicolumn{7}{|c|}{$\begin{array}{c}\text { Unidades que únicamente funcionan como prefijos en su lengua de } \\
\text { origen }\end{array}$} \\
\hline & $\begin{array}{c}D A \\
(1726-39)\end{array}$ & $\begin{array}{c}D R A E \\
(1803)\end{array}$ & $\begin{array}{c}D R A E \\
(1884)\end{array}$ & $\begin{array}{c}D R A E \\
(1925)\end{array}$ & $\begin{array}{c}D R A E \\
(1970)\end{array}$ & $\begin{array}{c}D R A E \\
(2001)\end{array}$ \\
\hline $\begin{array}{c}b i- \\
\text { (Del } \\
\text { lat. } b i-\text { ) }\end{array}$ & \multicolumn{4}{|c|}{$\varnothing$} & $\begin{array}{l}\text { preposición } \\
\text { inseparable } \\
\text { (sin guion) }\end{array}$ & $\begin{array}{l}\text { elemento } \\
\text { compositivo }\end{array}$ \\
\hline $\begin{array}{l}\text { tetra- } \\
\text { (Del gr. } \\
\tau \varepsilon \tau \rho \alpha ́-)\end{array}$ & \multicolumn{5}{|c|}{$\varnothing$} & $\begin{array}{l}\text { elemento } \\
\text { compositivo }\end{array}$ \\
\hline
\end{tabular}

Prefijos cuantificativos, asociados con la noción de cantidad o número ('dos' y 'cuatro', respectivamente)

$B i$ - se codifica a partir del $D R A E$ (1956), sin guion y bajo la etiqueta "preposición inseparable". Del mismo modo se recoge en 1970. En el DRAE (1984) se incorpora el guion y se modifica de categoría, pasando a llamarse "prefijo". En 1992, vuelve a cambiar de categoría, "elemento compositivo".

Por su parte, tetra- se recoge a partir del DRAE (1984). Desde su entrada al diccionario es denominado "elemento compositivo" y también se codifica seguido de guion.

Como en los casos de anti-, contra- y equi-, iso-, llama la atención que $b i$ - y tetra- presenten una tratamiento lexicográfico tan heterogéneo aun teniendo el mismo origen categorial y semántico. No obstante, ambos quedan denominados bajo el término "elemento compositivo" en el DRAE (2001).

En definitiva, mediante la revisión del tratamiento de distintos prefijos de igual naturaleza etimológica en el diccionario académico, hemos observado tres tendencias:

i. Circum- y peri-: prefijos con idéntica evolución lexicográfica, pero con distinta meta categorial ("prefijo" y "elemento compositivo", respectivamente).

ii. Equi-, iso-y bi-, tetra-: prefijos con distinta trayectoria en los diccionarios consultados, si bien los cuatro presentan la misma categoría en el DRAE (2001), "elemento compositivo".

iii. Anti- y contra-: prefijos que no comparten ni un tratamiento lexicográfico común a través de los siglos ni categoría gramatical final ("prefijo" y "prep. tiene uso como prefijo", respectivamente). 
En cualquier caso, comprobamos la falta de sistematicidad a la hora de incluir los prefijos en la macroestructura, así como de asignarles categoría gramatical. A continuación, examinamos el tratamiento ofrecido a los prefijos en la obra gramatical de la Corporación para comprobar, entre otras cuestiones, si existe la heterogeneidad documentada en los repertorios lexicográficos.

\section{TRATAMIENTO DE LOS PREFIJOS EN LAS GRAMÁTICAS ACADÉMICAS}

\subsection{BREVE REVISIÓN HISTÓRICA: DE LA GRAE (1771) AL EsBozo (1973)}

Una vez analizado el tratamiento categorial de los prefijos en la tradición lexicográfica académica, seguidamente nos proponemos revisar brevemente la teoría sobre los prefijos del español incluida en la Gramática de la Academia. No en vano, según afirma Alvar Ezquerra (1997: 128-129):

En el diccionario está continuamente presente la gramática, y ello es así porque no se puede aislar el léxico de la gramática, de la morfosintaxis. Es más, la lexicografía no es una disciplina independiente, al menos como práctica, sino que hace uso de las otras ramas de la lingüística: gramática y diccionario se complementan, se entrecruzan necesariamente, tanto en su forma como en sus contenidos, debido al carecer descriptivo que poseen, y a las necesidades didácticas que han de atender.

En lo que respecta al lugar que ocupa la prefijación en las $G R A E$, tras analizar una selección bastante representativa (en total, 13 textos: 1771, 1796, 1854, 1858, 1870, 1874, 1880, 1906, 1911, 1917, 1920, 1931 y Esbozo de una nueva gramática de la lengua española, 1973), observamos dos cuestiones importantes ${ }^{4}$ :

\footnotetext{
4 Ya en nuestra tesis doctoral revisamos el tratamiento de la prefijación en diccionarios y gramáticas publicados a lo largo de los siglos XVIII-XX, adscritos tanto a la corriente académica como a la no académica (vid. Torres 2009).
} 
i. En general, las gramáticas académicas publicadas entre 1771 y 1917 ubican la información concerniente a los procedimientos de formación de palabras y a los morfemas que intervienen en ellos en los capítulos dedicados al nombre (sustantivo y adjetivo), al verbo y a la preposición ${ }^{5}$.

ii. A partir de la edición de 1920, la Academia incluye como novedad en su Gramática un apartado sobre formación de palabras ("De la formación de palabras. La derivación, la composición y la parasíntesis") ${ }^{6}$, que se mantiene hasta la GRAE (1931).

En este capítulo sobre formación de palabras se detalla la lista de "prefijos empleados en la composición y parasíntesis", unidades que suelen ser "preposiciones o voces que solamente como tales prefijos tienen en castellano uso y valor" (abs-traer, di-sentir, mono-manía, proto-notario):

a- o an-, ab-, ad-, ana-, anfi-, anti-, archi-, bis- o biz-, cata-, centi-, circun-, cis-, citra-, deca-, deci-, des-, di-, dis-, en-, epi-, equi-, es-, ex-, extra-, hecto-, hiper-, hipo-, in-, inter-, kili-, meta-, mili-, miria-, mono, ob-, para-, per-, peri-, pos-, pre-, preter-, pro-, proto-, re-, res-, sin-, sub-, super-, trans- y ultra- (GRAE 1920: 165)

A la vista de la información morfológica contenida en la $G R A E$ (1920), podemos concluir que:

i. La Academia engloba la prefijación dentro de la composición.

ii. Bajo la categoría "prefijo" se incluyen tanto las "preposiciones" (esto es, prefijos que coinciden formalmente con preposiciones en español)

5 En concreto, acerca del tratamiento de la formación de palabras en gramáticas del siglo XIX, vid. Torres (en prensa).

6 Es necesario destacar la similitud de la teoría contenida en este nuevo capítulo con las esbozadas por José Alemany Bolufer en su Tratado de formación de palabras (1920). Esta influencia se hace extensiva a la mayoría de los pasajes sobre derivación, composición y parasíntesis incluidos en este capítulo de la GRAE (1920). No en vano hay que recordar dos hechos relevantes: (i) Alemany fue académico desde 1909 hasta 1934, año de su muerte, y (ii) su Tratado, antes de publicarse en libro (1920), vio la luz en el BRAE a modo de fascículos (1917-1919). De hecho, se ha adjudicado a Alemany la autoría del capítulo sobre formación de palabras incluido a partir de la GRAE (1920), a pesar de que las tareas encaminadas a la elaboración de la Gramática académica eran repartidas bajo el anonimato (vid. Sarmiento 2004). 
como las "voces que solo se usan como prefijos en castellano" (esto es, prefijos que no coinciden formalmente con preposiciones del español y que, por tanto, solo tienen un empleo ligado).

iii. Así, comprobamos que en la lista de "prefijos" se enumeran unidades que en los diccionarios son definidas como "preposición inseparable" (anti-, circum-, peri-). No en vano el término "prefijo" en la GRAE (1920) no se debe entender tal como lo interpretamos actualmente, sino como una macrocategoría en la que caben distintos tipos de unidades, entre las que se encuentran las preposiciones.

Por otro lado, en el Esbozo de una nueva gramática de la lengua española (1973), "un mero anticipo provisional de lo que será la nueva edición de la Gramática de la Lengua Española" (Esbozo 1973: 5), las cuestiones relativas a la formación de palabras se localizan únicamente en una sección preliminar denominada "Morfología. Generalidades". No obstante, en la "Advertencia" al Esbozo (1973: 6), al plantear las cuestiones de Morfología", se promete para la próxima edición de la gramática una novedad: un capítulo de formación de palabras que, según la Academia, "será objeto de publicación aparte y después se incorporará al texto definitivo de la Gramática"».

En lo que respecta a la teoría expuesta en el Esbozo sobre formación de palabras y, en concreto, sobre prefijación, destacamos las siguientes ideas:

i. Los "prefijos" no siempre son "morfemas trabados", ya que "las preposiciones propias pueden entrar en la formación del verbo $\mathrm{y}$, al mismo tiempo, regir nombres o pronombres" (en el caso de con-/ con, contribuir y con él, vid. Esbozo 1973: 170). Esta afirmación revela que, al igual que en las gramáticas académicas publicadas en los siglos XVIII, XIX y XX, en el Esbozo se identifican prefijos

7 Es necesario señalar que el gramático encargado de elaborar la parte sobre Morfología contenida en el Esbozo fue Salvador Fernández Ramírez. Como trabajos previos a la publicación académica de 1973, contamos con la ponencia pronunciada en el IV Congreso de Academias (1964) y con un artículo, titulado "Anticipos de la nueva Gramática” y publicado en el Boletín de la Real Academia Española (1968).

8 Fernández Ramírez únicamente redactó de manera parcial el apartado correspondiente a la derivación nominal, si bien contaba con abundantes datos, e incluso, escribió algunas páginas sobre composición, prefijación y parasíntesis, tal como indica Bosque (1986: 13). Este material quedó publicado en 1986 bajo el título La derivación nominal, ordenado, anotado y dispuesto para la imprenta por Ignacio Bosque. 
y preposiciones9. De hecho, se indica que, según el "método tradicional", se consideran "prefijos" tanto las "preposiciones propias" (ante-cámara, contra-hacer, sin-razón) como las "preposiciones impropias" (hiper-democracia) $)^{10}$.

ii. La explicación acerca de los "prefijos" no se ubica junto a la de los sufijos, esto es, bajo el epígrafe "morfemas derivativos", sino en el apartado denominado "composición", por lo que inferimos que la Academia sigue incluyendo la prefijación dentro de la composición. En consecuencia, observamos que el Esbozo (1973: 170), refiriéndose de manera explícita al "método tradicional", engloba dentro de la categoría de "prefijo" tanto a las "preposiciones propias" como a las "preposiciones impropias", anteriormente denominadas "preposiciones separables" - con significado dentro y fuera de la "composición"y "preposiciones inseparables" -que únicamente se emplean dentro de la "composición"-, respectivamente ${ }^{11}$.

iii. En cuanto a la categoría "elemento compositivo", hemos comprobado que no queda documentado en el Esbozo, publicado en 1973, si bien este término se empieza a usar en los diccionarios académicos a partir del DRAE (1970).

\footnotetext{
9 Actualmente, la delimitación preposición/prefijo ha sido objeto de estudio de numerosos autores, que adoptan las dos posturas documentadas desde antiguo: de una parte, algunos especialistas han defendido la identificación entre preposición y prefijo (Pottier 1962 y 1972; López 1970; Quilis 1970; Urrutia 1972; Moreno de Alba 1996, entre otros); de otra parte, diversos estudiosos cuestionan tal igualación y optan por no equiparar ambas unidades (Montero Curiel 1999; Varela y Martín García 1999; Rodríguez Ponce 2002; Felíu 2003, entre otros).

10 Cabe destacar que en el artículo del BRAE (1964), Fernández Ramírez no emplea la etiqueta de "preposiciones impropias", sino la denominación de "preverbios" (des-tiempo, re-cambio, hiper-democracia).

11 En este sentido, Gómez Asencio (1981: 254) señala que la mayoría de los autores adscritos a la tradición gramatical española identifica preposiciones y prefijos, pues distinguen dos tipos de preposiciones: "aisladas" y "en composición", denominadas más adelante "separables" e "inseparables" o "propias" e "impropias". En un trabajo posterior Gómez Asencio (1985: 174) vuelve a insistir en que "algunos autores incurrían en contradicción con la definición que daban para preposición: si preposición es la palabra que expresa una relación entre dos ideas o cosas, los prefijos (preposiciones impropias para estos gramáticos) no podrán ser considerados como preposiciones, ya que no tienen cabida en la definición”.
} 


\subsection{PREFiJo VS. ELEMENTO COMPOSITIVO EN LA NuEVA GRAMÁTICA DE LA RAE}

Como es sabido, tras casi cuatro décadas de espera, la nueva gramática académica ha visto la luz a finales de $2009 \mathrm{y}$, entre otros, sus objetivos son describir las construcciones gramaticales propias del español general, registrar las variantes conversacionales documentadas en la lengua no estándar, así como responder a las posibles dudas sobre cuestiones normativas.

En definitiva, la Nueva gramática se concibe como un texto de carácter normativo y, a la vez, descriptivo. En lo que respecta a su disposición, la obra se articula en tres partes fundamentales:

I. Cuestiones generales, donde se describen las partes de la gramática, las relaciones entre ellas y las unidades fundamentales del análisis gramatical.

II. Morfología, que analiza la estructura interna de las palabras.

III. Sintaxis, que analiza las estructuras que se crean en función de la forma en que se ordenan y se combinan las palabras.

Dentro de la Morfología, la prefijación cuenta con un capítulo, en concreto el diez. El once se dedica a la composición, dato mediante el que inferimos que la prefijación ya no se engloba dentro de la composición, a diferencia de los textos gramaticales publicados anteriormente por la Academia (GRAE 1771-1931 y Esbozo 1973).

En lo que respecta a la delimitación entre "prefijo" y "elemento compositivo", a diferencia del diccionario académico, encontramos abundante información que nos aclara el estatuto de ambas unidades:

i. Definición. En el capítulo sobre prefijación se alude explícitamente a la delimitación entre "prefijo" y "elemento compositivo". El prefijo se define como el "morfema que precede a la base léxica" (des-, in-, pre-, micro-) y el elemento compositivo queda caracterizado como la "base léxica grecolatina que posee un estatuto intermedio entre las formas ligadas y las libres" (filo-, logo-, helio-). En la NGRAE los prefijos no solo se definen mediante su posición (parámetro empleado también en el diccionario), sino también mediante otros criterios: clase de palabra a la que se asimilan; dependencia o independencia formal; significado y relación con las propiedades sintácticas de los predicados. Además, cabe destacar que ya no se equiparan prefijos y preposiciones, tal como ocurría en los textos gramaticales académicos publicados con anterioridad. 
ii. Adscripción a la derivación/composición. Las palabras creadas mediante prefijos ya se consideran derivadas en la NGRAE, mientras que aquellas formadas a partir de elementos compositivos se engloban bajo la composición:

Debido a que a veces pueden constituir el segundo segmento de una unidad léxica compleja (germanófilo, filólogo), estos componentes se asimilan a las formas compuestas, en lugar de a las prefijadas. De este modo, es hoy mayoritario, el análisis de estas unidades como temas neoclásicos que constituyen elementos compositivos (NGRAE 2009: 664).

No obstante, en la nueva edición de la gramática se plantea el hecho de que algunas unidades, consideradas elementos compositivos en la tradición gramatical y lexicográfica, actualmente se reconocen como prefijos:

Sin embargo, se suelen analizar hoy como prefijos otras unidades que tradicionalmente se consideraban elementos compositivos, por tanto el primer segmento de una palabra compuesta: ante-, vice-, mono-, semi-, sobre-, etc. (NGRAE 2009: 664).

iii. Gramática vs. Diccionario. A fin de justificar la falta de acuerdo entre gramática y diccionario en la propia Corporación, en la $N G R A E$ (2009: 664) se plantea la siguiente salvedad: "debe tenerse en cuenta que los criterios aplicados en este ámbito de la gramática no siempre coinciden con los que se usan en lexicografía". Efectivamente, como bien se explica en la nueva gramática, aunque sirven al usuario en el conocimiento de la lengua, ambas disciplinas poseen objetivos y metas diferentes:

En esta última disciplina [la lexicografía] suele otorgarse mayor peso a la etimología de la voz, así como a las palabras, a menudo independientes, con las que puede emparentarse históricamente. En los estudios de gramática sincrónica se presta más atención, en cambio, a las propiedades combinatorias de cada segmento y a la relación que cabe establecerse con su base morfológica. Del distinto valor concedido a cada uno de estos criterios surgen, en ocasiones, discrepancias en la clasificación que desde cada una de estas disciplinas se hace de los prefijos del español (NGRAE 2009: 664).

iv. ¿Prefijo o elemento compositivo? La NGRAE plantea el hecho de que algunas unidades pueden presentar un doble análisis, esto es, 
cabe la posibilidad de que sean consideradas prefijos o elementos compositivos $^{12}$ :

La diferencia entre prefijo y base compositiva (o elemento compositivo) es insegura en un buen número de casos: auto-, hiper-, macro-, micro-, mono-, multi-, poli-, etc. De hecho, estas formas se consideran elementos compositivos en unos análisis y prefijos en otros. Ambas opciones están justificadas, ya que los límites entre composición y prefijación afectan precisamente a estas unidades [...] En general, en la polémica acerca de si los segmentos mencionados deben considerarse parte de la composición o de la derivación pesa mucho la relevancia que se dé a los dos criterios fundamentales que intervienen: etimología y estructura morfológica de los derivados (NGRAE 2009: 664).

Efectivamente, según hemos intuido más arriba al analizar la categoría gramatical asignada a los prefijos en los diccionarios, en el nuevo texto gramatical de la Academia se indica que habitualmente la decisión de hablar de prefijo o elemento compositivo viene motivada por el origen etimológico categorial de la unidad en cuestión: "entienden algunos autores que serían prefijos los segmentos de origen preposicional (intra-, super-, ante-, peri-), pero no los de origen adjetival (neo-, pseudo-, paleo-, micro-)", si bien se reconoce igualmente "que unos y otros constituyen raíces en latín o en griego, por lo que la distinción no está justificada si se plantea en términos categoriales" (NGRAE 2009: 664).

Además, se propone el hecho de que el estatuto de los elementos objeto de estudio quede decidido con base en otros criterios, tales como la productividad que posean en nuestra lengua, su idiosincrasia etimológica o su semántica:

Se ha aducido asimismo que la distinción debe establecerse en función del rendimiento que ofrezcan en español, de sus propiedades gramaticales en la lengua de origen, o bien de la naturaleza más o menos abstracta de su significado. En varios sistemas terminológicos han sido llamados falsos prefijos, cuasiprefijos, prefijoides o prefijos impropios. Otros autores han acuñado términos híbridos como el de raices compositivas prefijas (NGRAE 2009: 664).

12 Sobre el estatuto y la denominación de los llamados elementos compositivos, véanse las consideraciones teóricas propuestas realizadas por algunos lingüistas como Alba de Diego (1983), Guerrero Ramos (1995), Rebollo (1997), García Platero (1998), Val Álvaro (1999), Varela y Martín García (1999), Stehlík (2001), Varela (2005). 
De este modo, la solución que propone la Academia consiste en aceptar el doble análisis cuando se plantee dificultad al determinar la naturaleza del elemento en cuestión.

Se analizarán aquí estos segmentos junto con los demás prefijos y se presentarán agrupados con otros que pertenecen a paradigmas similares en función de su significado. No obstante, tal como se ha señalado, se considera que está también justificada la opción de considerarlos segmentos compositivos de origen culto. Como se explicará, algunos poseen gran productividad en la lengua común, mientras que otros la manifiestan solo en el lenguaje de la ciencia y de la técnica o en otros dominios restringidos del léxico (NGRAE 2009: 665).

No obstante, en la Nueva gramática se aclaran casos en los que elementos de distinta índole confluyen en una misma forma. Se trata de la confusión que ocasiona la coincidencia formal entre algunos prefijos o temas grecolatinos con elementos procedentes de acortamientos modernos que participan en procesos de formación de palabras anteponiéndose a una palabra independiente ${ }^{13}$ :

Se distinguirá aquí el prefijo auto- (autocrítica, autodefensa) del segmento compositivo auto-, resultado del acortamiento de la palabra automóvil: autobús, autocaravana, autocarril, autocine, autoescuela, etc. De manera análoga, en el grupo de prefijos de sentido espacial se ha incluido tele-. En este uso, tele- equivale a 'distancia', como en telebanco, telecabina, telecomunicación [...] En cambio este mismo segmento constituye un acortamiento del sustantivo televisión en telebasura, telecomedia, telediario

13 En efecto, si establecemos una comparación entre los acortamientos y los temas grecolatinos, se observa una serie de relaciones y diferencias entre ambas unidades (vid. Casado, 1999: 5077-5080 y Varela, 2005: 89-92). De una parte, entre las similitudes entre los acortamientos y los temas cultos destacan su fonología (bisílabos de acentuación llana), su morfología (participan en procesos compositivos y tienen generalmente libertad posicional), su semántica (se trata de unidades léxicas de contenido básico general -estable y no relacional, que los diferencia de los afijos) y su forma (se documenta homonimia en casos como auto/auto-, fotolfoto-, radio/radio-, tele/tele-). De otra parte, en cuanto a las diferencias existentes, encontramos aspectos morfológicos (los acortamientos no siempre coinciden con la frontera morfológica, por ejemplo, paraca o diver, procedentes de paracaidista y divertido, respectivamente) o sintácticos (los temas cultos no pueden aparecer en la sintaxis de forma aislada, ya que necesitan adjuntarse a afijos derivativos o lexemas para adquirir independencia sintáctica). 
y otras voces similares que se analizan como palabras compuestas (NGRAE 2009: 665$)^{14}$.

\section{VALORACIÓN FINAL}

Tras revisar el tratamiento de los prefijos en los diccionarios académicos, comprobamos que en el DRAE (2001) se emplean dos tipos de categorías a la hora de caracterizar estas unidades: "prefijo" y "elemento compositivo".

Aunque estos términos no se definen de manera clara y precisa ni en el diccionario ni en los textos gramaticales académicos publicados entre 17711973, en la Nueva gramática (2009) se atiende a tal delimitación y se justifica el hecho de emplear "prefijo" o "elemento compositivo" ("según la relevancia que se dé a los dos criterios fundamentales que intervienen: etimología y estructura morfológica de los derivados", NGRAE 2009: 664).

No obstante, a pesar de la posibilidad del doble análisis, pensamos que la Academia debería unificar la terminología mediante la que se refiere a las unidades que hemos estudiado en esta investigación, especialmente en sus repertorios lexicográficos.

En definitiva, hemos de pensar en el usuario que acude al diccionario y encuentra que circun- y peri-, a pesar de poseer idéntica etimología y significado, se definen como "elemento compositivo" y "prefijo", respectivamente.

No en vano, según afirma Alvar Ezquerra (1997: 128-129):

En el diccionario está continuamente presente la gramática, y ello es así porque no se puede aislar el léxico de la gramática, de la morfosintaxis. Es más, la lexicografía no es una disciplina independiente, al menos como práctica, sino que hace uso de las otras ramas de la lingüística: gramática y diccionario se complementan, se entrecruzan necesariamente, tanto en su forma como en sus contenidos, debido al carácter descriptivo que poseen, y a las necesidades didácticas que han de atender.

14 En un estudio de carácter más concreto, Felíu (2003) se centra en el tratamiento de unidades morfológicas como auto- y su distinto estatus en palabras como autoengaño y autocine, cuestión que refleja los problemas teóricos existentes en torno a la relación entre la composición, tanto española como grecolatina, y la prefijación. 


\section{REFERENCIAS BIBLIOGRÁFICAS}

Alba de Diego, Vidal. 1983. Elementos prefijales y sufijales: ¿derivación o composición? Serta Philológica Fernando Lázaro Carreter, I, pp. 17-21. Madrid: Cátedra.

Alvar Ezquerra, Manuel. 1993. La formación de palabras en español. Madrid: Arco/ Libros.

1997. Algunos aspectos de la presencia gramatical en el diccionario. En José Manuel González y Jesús Terrón (eds.). Actas de las IV Jornadas de metodología y didáctica de la lengua española, pp. 115-130. Cáceres: Universidad de Extremadura.

Bosque, Ignacio. 1986. Prefacio. La derivación nominal, Boletín de la Real Academia Española, anejo XL.

Casado Velarde, Manuel. 1999. Otros procesos morfológicos: acortamientos, formación de siglas y acrónimos. En Ignacio Bosque y Violeta Demonte (dirs.). Gramática descriptiva de la lengua española, III, pp. 5075-5096. Madrid: RAE-Espasa Calpe.

Comisión Permanente de la Asociación de Academias de la Lengua española. 1966. Memoria del IV Congreso de Academias de la Lengua Española (Buenos Aires, 30 de noviembre-10 de diciembre de 1964). Buenos Aires: Academia Argentina de las Letras.

Fernández Ramírez, Salvador. 1964. Para la futura Gramática. Boletín de la Real Academia Española, XLIV, cuaderno CLXXIII, pp. 431-448.

1986. La derivación nominal, Boletín de la Real Academia Española, anejo XL. [Volumen ordenado, anotado y dispuesto para la imprenta por Ignacio Bosque].

Felíu Arquiola, Elena. 2003. Morfología derivativa y semántica léxica: la prefijación de auto-, co- e inter-. Madrid: Ediciones de la Universidad Autónoma de Madrid.

García Platero, Juan Manuel. 1998. Los seudoprefijos. Su importancia para la creación léxica. En Feliciano Delgado León, M. ${ }^{a}$ Luisa Calero Vaquera y Francisco Osuna García. Estudios de Lingüística general, Actas del II Simposio de Historiografía Lingüística, pp. 291-301. Córdoba: Ediciones de la Universidad.

Gómez Asencio, José J. 1981. Gramática y categorías verbales en la tradición española (1771-1847). Salamanca: Universidad de Salamanca.

1985. Subclases de palabras en la tradición española (1771-1847). Salamanca: Universidad de Salamanca.

Guerrero Ramos, Gloria. 1995. Neologismos en el español actual. Madrid: Arco/Libros.

López, M. ${ }^{a}$ Luisa. 1970. Problemas y métodos en el análisis de preposiciones. Madrid: Gredos.

Montero Curiel, M. ${ }^{\text {a }}$ LuISA. 1999. La prefijación negativa en español. Cáceres: Universidad de Extremadura.

Moreno de Alba, José. G. 1996. La prefijación en el español de México. México: Universidad Nacional Autónoma de México-Instituto de Investigaciones Filológicas.

Pottier, Bernard. 1962. Sistématique des éléments de relation. París: Libraire Klincksieck. 1972. Introduction a l'étude linguistique de l'espagnol. París: Ediciones Hispanoamericanas.

Quilis, ANTONio. 1970. Sobre la morfonología. Morfonología de los prefijos en español. Revista de la Universidad de Madrid, XIX, 74, pp. 223-248.

Rebollo Toríos, Miguel Ángel. 1997. Precisiones sobre los llamados temas. Anuario de Estudios Filológicos, XX, pp. 357-366.

Rodríguez Ponce, M. ${ }^{a}$ Isabel. 2002. La prefijación apreciativa en español. Cáceres: Universidad de Extremadura.

SARmiento, Ramón. 2004. Gramáticos anónimos en la Academia: un monumento perenne al hispanismo. En Cristóbal Corrales, Josefa Dorta, Antonia Nelsi, Dolores Corbella y 
Francisca del Mar Plaza (coords.). Nuevas aportaciones a la historiografia lingüistica. Actas del IV Congreso Internacional de la SEHL (La Laguna, Tenerife, 22-25 de octubre de 2003), II, pp. 1525-1539. Madrid: Arco/Libros.

Stehlíк, Petr. 2001. Elementos prefijales cultos: ¿morfemas compositivos o prefijos? Studia minora facultatis philosophicae universitatis brunensis, XXII, pp. 105-114.

Torres Martínez, Marta. 2008. Tratamiento de los elementos compositivos en la lexicografía académica y extraacadémica del siglo XX. En Dolores Azorín Fernández (dir.). El diccionario como puente entre las lenguas y culturas de mundo, Actas del II Congreso Internacional de Lexicografía Hispánica, pp. 393-401. Alicante: Universidad de Alicante (Taller digital)-Fundación Biblioteca virtual Miguel de Cervantes. 2009. La prefijación en gramáticas y diccionarios del español (siglos XVIII-XX). Jaén: Universidad de Jaén [Tesis doctoral inédita].

en prensa. Tratamiento de la formación de palabras en gramáticas del español del siglo XIX. Estudios de Lingüistica. Universidad de Alicante 24.

Urrutia CÁrdenas, Hernán. 1972. Análisis semántico-funcional de los prefijos. Estudios Filológicos, 10, pp. 291-335.

Val Álvaro, José Francisco. 1999. La composición. En Ignacio Bosque y Violeta Demonte (dirs.). Gramática descriptiva de la lengua española, III, pp. 4757-4841. Madrid: RAEEspasa Calpe.

Varela Soledad. 2005. Morfología léxica: la formación de palabras. Madrid: Gredos.

Varela, Soledad y Josefa Martín García. 1999. La prefijación. En Ignacio Bosque y Violeta Demonte (dirs.). Gramática descriptiva de la lengua española, III, 4993-5038. Madrid: RAE-Espasa Calpe.

CORPus

$D R A E=$ Real Academia de la Lengua Española. 2001 Diccionario de la lengua española. Madrid: Espasa Calpe.

GRAE = Real Academia de la Lengua Española. 1931. Gramática de la lengua española . Madrid: Espasa Calpe.

$N G R A E=$ Real Academia de la Lengua Española. 2009. Nueva gramática de la lengua española. Madrid: Espasa Calpe.

$E S B O Z O=$ Real Academia de la Lengua Española. 1973. Esbozo de una nueva gramática de la lengua española. Madrid: Espasa Calpe. 